\title{
First results from the UK superSTEM Laboratory
}

A. Bleloch*, L.M. Brown ${ }^{* *}$, R. Brydson ${ }^{* * *}$, A. Craven ${ }^{* * * *}$, U. Falke*, M. Falke*, P. Goodhew ${ }^{* * * * *}$ and G. Tatlock ${ }^{* * * * *}$

*SuperSTEM Laboratory, CLRC, Daresbury WA4 4AD, UK.

** University of Cambridge, Cavendish Laboratory, Cambridge CB3 0HE, UK

**** Materials Science, University of Leeds, Leeds, LS2 9JT, UK

${ }^{* * * *}$ Physics and Astronomy, The Kelvin Building, University of Glasgow, G12 8QQ

${ }^{* * * * *}$ Materials Science and Engineering, University of Liverpool, L69 3GH, UK

The first European aberration corrected STEM instrument was installed in its purpose built laboratory in November 2002. It is a VG Instruments HB501 fitted with an aberration corrector supplied by Nion Co. The initial performance of the instrument and the building is illustrated here with examples of problems addressed by high angle annular dark field (HAADF) imaging. These examples include; a semiconductor interface structure between silicon and nickel silicide[1] and an InGaN single quatum well in $\mathrm{GaN}$ with indications of the variation of the starting and finishing interface[2]. As a benchmark, an atomic resolution HAADF image of diamond [3] with a spatial resolution of $0.108 \mathrm{~nm}$ in all directions with a $100 \mathrm{keV}$ beam is also shown.

In electron optics, spherical aberration correction has been a practicable reality for almost half a decade[4,5]. At this stage, it is important that the early adopters of the technique to demonstrate the scientific effectiveness of the new technology on a variety of systems. One emphasis we wish to place on these data is the relatively modest time and effort from specimen insertion to interpretable conclusion. For example, the work on the interface between silicon and nickel disilicide took a total of four days of microscope time to yield an atomic model of the $2 x 1$ interface reconstruction without recourse to simulation. It goes without saying that this proposed model requires further investigation not least using theoretical codes but the quality of the information from the ADF images significantly reduces the search space of parameters when compared with a high resolution electron microscopy (HREM) approach. It should also be mentioned that the quantum well image shown in figure 1 is the result of one day on the microscope with a well-prepared sample. The interface roughness can be seen on an atom column by atom column scale.

A spatial resolution of at least $0.108 \mathrm{~nm}$ ((311) spacing in HAADF images of diamond) has been easily accessible at each time of asking with the $100 \mathrm{keV}$ electron probe as shown in figure 2 . The optimum operating mode, particularly with reference to source demagnification and beam diameter within the corrector, has not yet been experimentally ascertained largely because it is dependent on the detailed influence of environmental noise and instabilities. All of these influences are constantly being improvement. Simultaneous electron energy loss spectroscopy at $0.5 \mathrm{eV}$ spectral resolution has been achieved but is not shown here.

In figure 3 the silicon dumbbells can be easily resolved in the silicon region to the right. The bright contrast from the nickel shows that the interface is atomically sharp although there is an indication of some variation through the sample in the lower half of the right hand image. The nickel occupancy of the columns and their position shows a $2 \mathrm{x}$ reconstruction on the right and $1 \mathrm{x}$ for the orthogonal view on the left[6] 


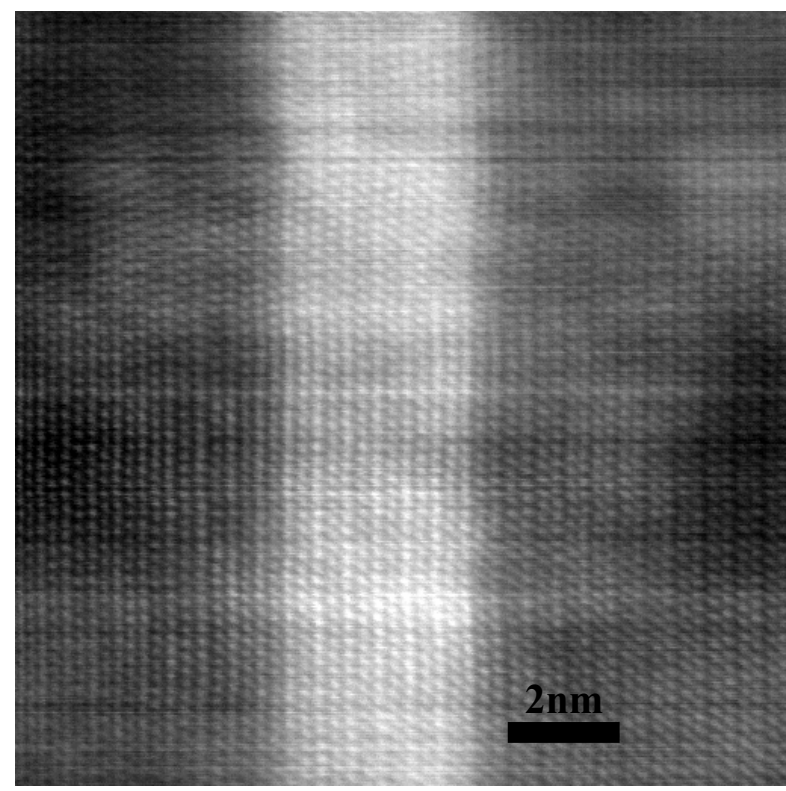

FIG. 1. An InGaN quantum well in GaN grown along the c-axis from right to left. Zcontrast allows a detailed evaluation of interface roughness.

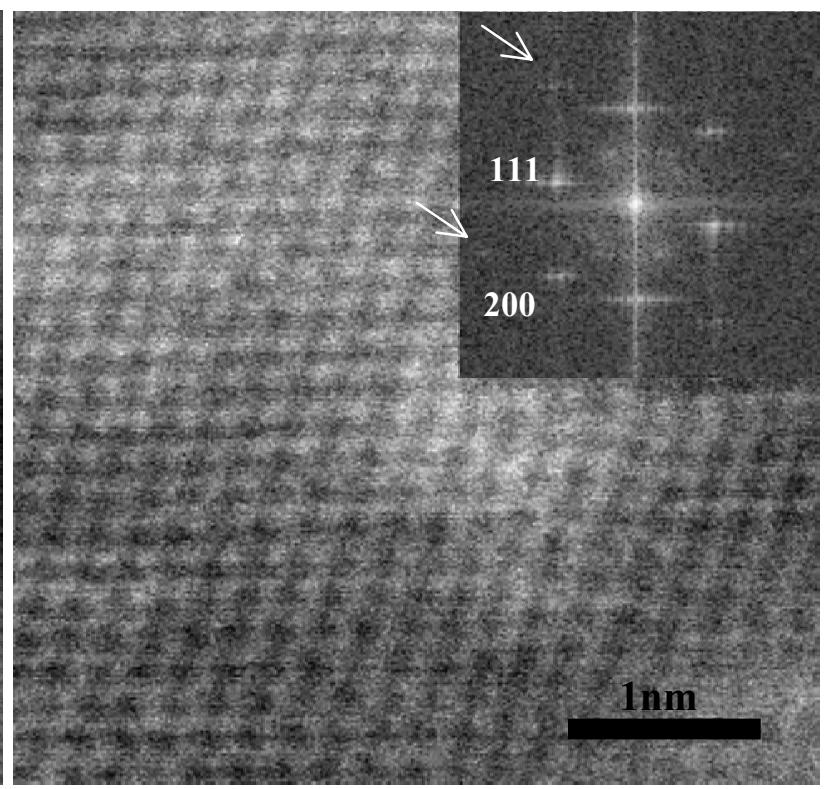

FIG. 2. The diamond lattice is a stringent test of HAADF imaging because of the low atomic number. The 311 type reflections $(0.108 \mathrm{~nm})$ are visible (arrowed) in two directions.
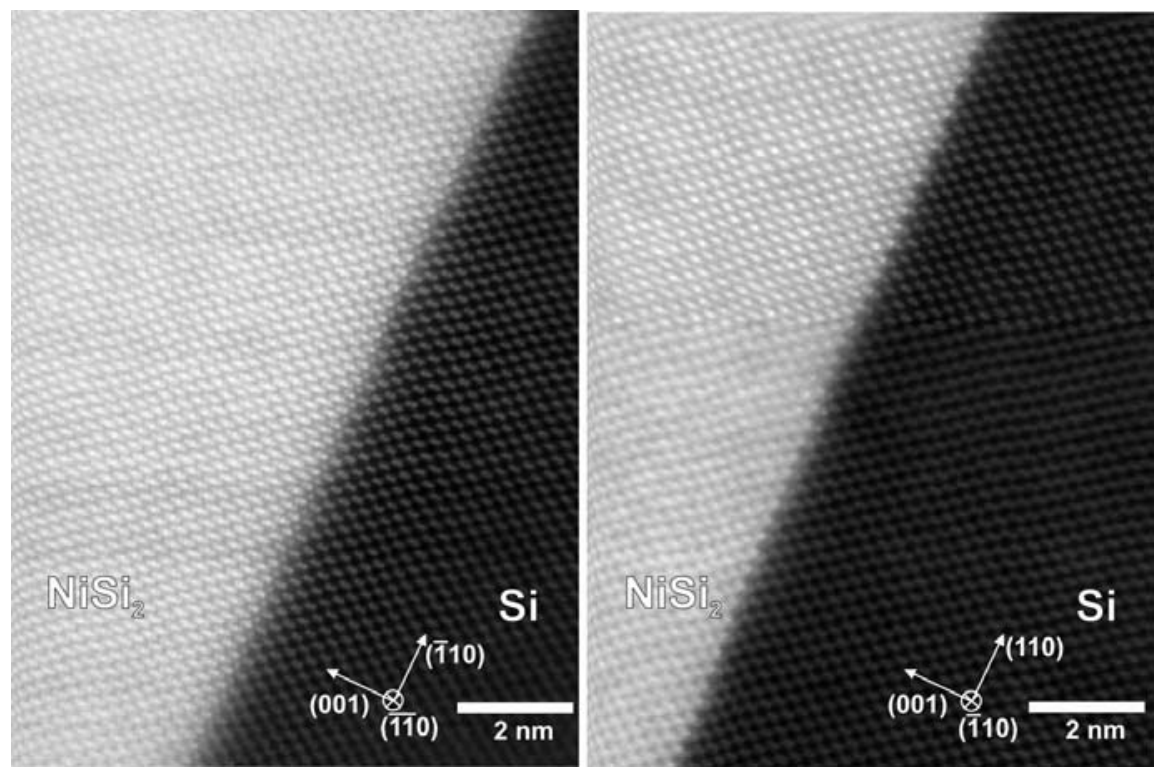

FIG. 3. Two orthogonal views of the 100 interface between nickel disilicide and silicon

References and acknowledgements:

1 Sample from St. Teichert, G. Beddies, H.-J. Hinneberg, Insitute of Physics, TU-Chemnitz 2 Sample from T. Smeeton, Dr. J Barnard and Prof. C. Humphreys, Cambridge University. 3 Support and sample from DTC, UK

4 O. L. Krivanek, N. Delby and A. R. Lupini, (1999), Ultramicroscopy, 78, 1-11

5 M. Haider et al., (1998), Nature, 392, 768

6 We would like to thank the EPSRC, CLRC Daresbury, EMAG (IOP), and the RMS 